\title{
A Genuinely Short Gamma-Ray Burst from Massive Star Core Collapse
}

Binbin Zhang ( $\sim$ bbzhang@nju.edu.cn )

Nanjing University https://orcid.org/0000-0003-4111-5958

Zi-Ke Liu

Nanjing University

Zong-Kai Peng

Nanjing University

Ye Li

Peking University

H.J. Lu

GXU

Jun Yang

Nanjing University

Yi-Si Yang

Nanjing University

Yu-Han Yang

Nanjing University https://orcid.org/0000-0003-0691-6688

Yan-Zhi Meng

Nanjing University

Jin-Hang Zou

Nanjing University

Hao-Yang Ye

Nanjing University

Xiang Wang

Guangxi University

Jirong Mao

Yunnan Observatories, Chinese Academy of Sciences

Xiao-Hong Zhao

Yunnan Observatory

Jinming Bai

Yun Nan Astronomical Observatory, Chinese Academy of Sciences

Alberto Castro-Tirado

Instituto de Astrofísica de Andalucía-CSIC https://orcid.org/0000-0003-2999-3563

Y.D. Hu 
Instituto de Astrof \'isica de Andalucl'ia (IAA-CSIC), Glorieta de la Astronom \'ia

\section{Zi-Gao Dai}

Nanjing University

Bing Zhang

University of Nevada, Las Vegas https://orcid.org/0000-0002-9725-2524

\section{Physical Sciences - Article}

Keywords: gamma-ray bursts, GRB, star core collapse

Posted Date: December 31st, 2020

DOl: https://doi.org/10.21203/rs.3.rs-131126/v1

License: (c) (1) This work is licensed under a Creative Commons Attribution 4.0 International License. Read Full License

Version of Record: A version of this preprint was published at Nature Astronomy on July 26th, 2021. See the published version at https://doi.org/10.1038/s41550-021-01395-z. 


\section{A Genuinely Short Gamma-Ray Burst from Massive Star Core Collapse}

B.-B. Zhang ${ }^{1,2,3 \dagger}$ Z.-K. Liu ${ }^{1,2}$, Z.-K. Peng ${ }^{1,2}$, Y. Li ${ }^{4}$, H.-J. Lü ${ }^{5}$, J. Yang ${ }^{1,2}$, Y.-S. Yang ${ }^{1,2}$, Y.-H. Yang $^{1,2}$, Y.-Z. Meng ${ }^{1,2}$, J.-H. Zou ${ }^{1,2,6}$, H.-Y. Ye ${ }^{1}$, X.-G. Wang ${ }^{5}$, J.-R. Mao ${ }^{7}$, X.-H. Zhao ${ }^{7}$, J.-M. Bai $^{7}$, A. J. Castro-Tirado ${ }^{7,8}$, Y.-D. Hu ${ }^{7,8}$, Z.-G. Dai ${ }^{1,2}$, B. Zhang ${ }^{3 \ddagger}$

${ }^{1}$ School of Astronomy and Space Science, Nanjing University, Nanjing 210093, China

${ }^{2}$ Key Laboratory of Modern Astronomy and Astrophysics (Nanjing University), Ministry of Education, China

${ }^{3}$ Department of Physics and Astronomy, University of Nevada, Las Vegas, NV 89154, USA

${ }^{4}$ Kavli institute for astronomy and astrophysics, Peking University, Beijing. 100871, P.R.China

${ }^{5}$ Guangxi Key Laboratory for Relativistic Astrophysics, School of Physical Science and Technology, Guangxi University, Nanning 530004 China;

${ }^{6}$ College of Physics, Hebei Normal University, Shijiazhuang 050024, China

${ }^{7}$ Yunnan Observatories, Chinese Academy of Sciences, 650216, Kunming, China

${ }^{8}$ Instituto de Astrofísica de Andalucía (IAA-CSIC), Glorieta de la Astronomía s/n, E-18008, Granada, Spain

${ }^{9}$ Departamento de Ingeniería de Sistemas y Automática, Escuela de Ingenierías, Universidad de Málaga, Dr. Pedro Ortiz Ramos, 29071 Málaga, Spain

Gamma-ray bursts (GRBs) have been phenomenologically classified into long and short populations based on whether the observed duration is longer or shorter than two seconds ${ }^{\mathrm{I}}$. Multi-wavelength and multi-messenger observations in recent years have revealed that in general long GRBs originate from massive star core collapse events ${ }^{2}$, whereas short GRBs originate from binary neutron star mergers ${ }^{3}$. It has been known that the duration criterion is sometimes unreliable, and multi-wavelength criteria are needed to identify the physical origin of a particular GRB ${ }^{4}$. Some apparently long GRBs have been suggested to have a neutron star merger origin ${ }^{5}$, whereas some apparently short GRBs have been attributed to genuinely long GRBs ${ }^{6}$ whose short, bright emission is above the detector's sensitivity threshold. Still, there has been no known case that a GRB is genuinely short but originates from death of a massive star. Here we report the comprehensive analysis of the multi-wavelength data of a bright short GRB 200826A. This burst has a sharp 1-second spike, which is not part of an underlying long-duration event. Its other observational properties are, however,

\footnotetext{
${ }^{\dagger}$ E-mail: bbzhang@nju.edu.cn,(10) orcid.org/0000-0003-4111-5958

${ }^{\ddagger}$ E-mail: zhang@ @hysics.unlv.edu,(1) orcid.org/0000-0002-9725-2524
} 
inconsistent with those of other short GRBs believed to originate from binary neutron star mergers. Rather, these properties resemble those of long GRBs. This burst presents a challenge to the traditional GRB classification scheme and reveals a class of core-collapse-origin GRBs with genuinly short durations.

GRB 200826A triggered the Fermi Gamma-ray Burst Monitor (GBM) ${ }^{\square}$ at 04:29:52 Universal Time on 26 August $2020^{8}$. Follow-up observations detected the optical counterpart ${ }^{\underline{Y}}$ and identified its host galaxy and redshift at $z=0.7481$ (Ref. [10). The 10-800 keV-band light curve, as detected by GBM, is shown in Figure Wa. The duration in the same energy range of the burst is measured $^{\mathbb{1}}$ as $T_{90} \sim 0.96_{-0.08}^{+0.05} \mathrm{~s}$ (see Table 1 for a collection of all the measured parameters of GRB 200826A, and see the details of data analysis in Methods). The lightcurve pulse profile is quite sharp. There is no signal above the background both before and after the burst, hinting that the short duration is genuine. We further measure the "amplitude parameter" of this burst $\mathrm{t}^{[2]}$, which is defined as the ratio between the peak flux and the average background flux of the GRB lightcurve (see Methods). We obtain $f=7.58 \pm 1.23$ for GRB 200826A. To make a long GRB a "tip-oficeberg" short GRB, the effective $f$ value is typically $<1.5$ (Ref. ए2). The disguised short GRB 090426 with a massive star origin ${ }^{6}$ had $f=1.48 \pm 0.11$, which is consistent with having underlying long-duration emission not observed above the detection level. The enormous $f$ value of GRB 200826A, therefore, suggests that it is a genuinely short GRB and cannot be the tip-of-iceberg of a long GRB (Figure.⿴lb).

Figure Wc shows the standard long/short classification diagram in the duration - hardness ratio domain, where the hardness ratio is defined as observed counts ratio between the 50-300 $\mathrm{keV}$ band and the 10-50 keV band within the $T_{90}$ duration (see Methods). One can see that GRB 200826A falls into the distribution of short GRBs, even though near the softer end of the hardness ratio distribution (with a value 0.803 ). We perform the spectral analysis of the GRB (see Fermi data analysis in Methods and Extended Data Table $\mathbb{1}$ ). Its time-averaged photon index is $\alpha=$ $-0.68 \pm 0.05$ and the spectral peak energy is $E_{\mathrm{p}} \simeq 120.29_{-3.67}^{+3.93} \mathrm{keV}$ (see also Extended Data Figure (2). These are not too different from other short GRBs. Without the redshift and host galaxy information, this burst would be classified as a member in the short GRB population.

The conclusion changes when the redshift information is considered. With the measured redshift, we derive an isotropic energy of $E_{\gamma \text {,iso }} \simeq 7.09 \pm 0.28 \times 10^{51}$ erg based on the measured fluence of $4.85 \pm 0.19 \times 10^{-6} \mathrm{erg} \mathrm{cm}^{-2}$. This energy value is relatively large compared with other short GRBs but is typical in the long GRB population. Such a discrepancy is more obvious when GRB 200826A is plotted in the $E_{\gamma, \text { iso }}-E_{\mathrm{p}, \mathrm{z}}$ relation for both long and short GRBs (where $E_{\mathrm{p}, \mathrm{z}}=$ $E_{\mathrm{p}}(1+z)$ is the spectral peak energy in the burst rest frame). Observations showed that there is an

${ }^{\text {i }}$ We note that Ref. ${ }^{\square}$ presents a slightly different value of $\mathrm{T}_{90}=1.14 \pm 0.13$ in 50-300 keV energy range. 
empirical relation between the two, the so-called Amati-relation ${ }^{[3]}$ (see Methods). Long and short GRBs are known to follow the same trend but form distinct tracks ${ }^{4}$. We find that GRB 200826A squarely lies on the long GRB track rather than on the short GRB track (Figure \a).

The peculiarity of GRB 200826A is also reflected in its value of the $\epsilon \equiv E_{\gamma, \text { iso, } 52} / E_{\mathrm{p}, \mathrm{z}, 2}^{5 / 3}$ parameter ${ }^{\sqrt{14}}$, where $E_{\gamma, \text { iso }, 52}$ is the isotropic gamma-ray energy in units of $10^{52} \mathrm{erg}$, and $E_{\mathrm{p}, z, 2}$ is $E_{p, z}$ in units of $100 \mathrm{keV}$. Following the convention of Ref. [5], hereafter we define Type I and Type II GRBs as those with a compact star merger origin and a massive star core collapse origin, respectively. It has been shown that GRBs can be more distinctly classified into their respective physical categories (Type I and Type II) in the $\epsilon-T_{90}$ space, with a separation line of $\epsilon \sim 0.03$ (Ref. ${ }^{\text {(4) }}$ ). We obtain $\epsilon=0.33$ for GRB 200826A and find that it statistically falls into the Type II rather than the Type I category (Figure $\Sigma \mathrm{b}$, see Methods).

Long GRBs usually show "spectral lags", with the softer emission arriving later than the harder emission ${ }^{\square 6}$. Short GRBs, on the other hand, typically show negligible lags ${ }^{[17}$. The (10$20) \mathrm{keV}$ to (250-300) keV spectral lag of GRB 200826A is $0.157 \mathrm{~s}$ (Extended Data Figure B, see Methods). This is at odds for short GRBs but is typical for long GRBs.

The host galaxy and the position (see Methods) of the GRB inside the galaxy often carry the clue regarding the physical origin of a GRB ${ }^{\square-20}$. We performed a follow-up observation of GRB 200826A using Gran Telescopio Canarias (GTC) and identified the host galaxy (Figure 3a) which was found to lie at redshift $0.7481 \pm 0.0003$ (ref. $\square$ ). We measured its half-light radius as $0.77 "$, corresponding to $5.7 \mathrm{kpc}$, in our GTC $r$-band image. The optical afterglow of GRB 200826A has a 0.35 " offset from the center of the host galaxy, which corresponds to $2.56 \mathrm{kpc}$. The normalized offset is $r=R_{\text {off }} / R_{50}=0.45$. We estimate the cumulative light fraction $F_{\text {light }}=0.79$. We also calculated the star formation rate (SFR; see Methods) to be $>1.36 M_{\odot} \mathrm{yr}^{-1}$ and specific star formation rate (sSFR) to be $>0.33 \mathrm{Gyr}^{-1}$. Our results are consistent with those in Ref ${ }^{\square}$. Binary neutron star mergers are the leading engines for short-duration GRBs. These mergers usually happen after a long delay since star formation so that the SFR and sSFR of their host galaxies are usually not high. Due to kicks at the births of neutron stars, these mergers also usually occur at a site far away from star formation regions, often in the outskirts or even outside of their host galaxies $^{\text {[1, }}$, In Figure Bb-e, we place GRB 200826A in the $r, F_{\text {light }}$, SFR, and sSFR distributions for long (Type II) and short (Type I) GRBs ${ }^{22}$. One can see that the host galaxy properties of GRB 200826A are more consistent with belonging to Type II rather than Type I.

To make our conclusion more quantitative, we apply the method of Ref. ${ }^{201}$ that classifies GRBs into physical categories using multi-wavelength observational data (see Methods). The logarithmic odds $\log O$ (II : I $)_{\text {host }}$ using host galaxy information only is 2.5 , indeed consistent with 
a Type II origin. When the prompt emission information is considered, if one ignores the $f$ information, the overall indicator $\mathcal{O}$, which is defined as the logarithmic probability ratio between Type II and Type I GRBs plus 0.7 and assigns Type II/I GRBs as positive/negative values, is 1.5. This places GRB 200826A into the Type II (massive star core-collapse) GRB category (Figure [3f). When the very large $f$ value ( $f=7.58 \pm 1.23$, a character of Type I GRBs) is added as one of the criteria, the method of Ref. ${ }^{\text {QII }}$ gives $\mathcal{O}=-3.1$, still placing GRB 200826A in the Type I category. This again indicates the uniqueness of GRB 200826A, which points towards a genuinely short burst with other properties consistent with being a massive-star-core-collapse event, so that the GRB classification scheme requires further refinements.

Finally, a Type II origin of GRB 200826 is further supported by the follow-up observations by Gemini-North 8-meter telescope ${ }^{\square}$, which revealed a possible optical bump at $25.45 \pm 0.15 \mathrm{mag}$ in $i$-band. This bump could be consistent with a supernova, a signature of a massive star core collapse event.

The fact that GRB 200826A is genuinely short but its many properties are inconsistent with being of a neutron star merger origin raises a great challenge in identifying its progenitor star. For an accretion-powered GRB engine, the shortest timescale to power a relativistic jet is defined by the free-fall time scale of the star, which reads ${ }^{23} t_{\mathrm{ff}} \sim\left(\frac{3 \pi}{32 \mathrm{G} \bar{\rho}}\right)^{1 / 2} \sim 210 \mathrm{~s}\left(\frac{\bar{\rho}}{100 \mathrm{~g} \mathrm{~cm}^{-3}}\right)^{-1 / 2}$, where $\bar{\rho}$ is the mean density of the accreted matter. One can see that for the typical density $\bar{\rho} \sim 100 \mathrm{~g} \mathrm{~cm}^{-3}$ of a massive star, the duration should be long. If the $\sim 1 \mathrm{~s}$ duration of GRB 200826A is indeed the total duration of the central engine, one immediately poses a lower limit on the density of the accreted materials, i.e. $\bar{\rho}>4.4 \times 10^{6} \mathrm{~g} \mathrm{~cm}^{-3} T_{90}^{-2}$. This would directly rule out any massive star progenitor with an accretion-powered engine.

There are two ways to get around this argument. The first possibility is indeed to introduce a compact object rather than a massive star at the central engine. One category of model invokes a white dwarf (WD) engine. Since single WDs cannot make GRBs, one may consider various binary mergers invoking WDs ${ }^{24,25]}$, e.g., WD-WD mergers, WD-NS mergers, and WD-BH mergers. One challenge of this scenario is whether such GRBs can still occur in star-forming regions in the host galaxies. Moreover, the detection of a possible supernova bump ${ }^{\square}$ would disfavor this model. The other possible channel is that GRB 200826A's progenitor was a massive star, but it collapsed some time before the GRB was made. Such a scenario, known as "supranova", has been discussed in the literature ${ }^{26}$. The GRB is produced by the implosion of a supermassive neutron star, probably triggered by the spindown or fallback accretion of the neutron star. As the GRB jet is launched, there are no more low-density materials surrounding the source, so that the duration is short. The source is still in the star forming region. One challenge of this model is to account for the relatively 
shallow decay in the X-ray afterglow, which seems to require additional energy injection during the afterglow phase (Methods). The possible supernova bump ${ }^{\square}$ also requires that the delay time from massive star core collapse to GRB jet launching cannot be much longer than a day.

The second possibility is that the true duration of the central engine is long. However, during the majority of the active central engine time, the $\gamma$-ray emission is below the detection threshold of GRB detectors. Such a "tip-of-iceberg" interpretation may apply to other short-duration, low- $f$ Type II GRBs such as GRB 090426 (Refs. ${ }^{6}$ and ${ }^{[2}$ ). However, the large $f$ value of GRB 200826A makes this simple interpretation unlikely. One may consider two modified scenarios. One is that the total central engine timescale $\Delta t_{\text {eng }}$ is long. However, the majority of the time is used for the jet to penetrate through the stellar envelope in a timescale $\Delta t_{\text {jet }} \sim 10 \mathrm{~s}$. The observed GRB duration is $\Delta t_{\mathrm{GRB}}=\Delta t_{\text {eng }}-\Delta t_{\text {jet }}$, which could be as short as $\sim 1 \mathrm{~s}$ (Ref. ${ }^{27}$ ). Within this interpretation, one needs to introduce a coincidence between $\Delta t_{\text {eng }}$ and $\Delta t_{\text {jet }}$. This possibility has been emphasized also in ref. ${ }^{\square}$. Alternatively, one may envisage that the engine indeed lasted a duration much longer than $1 \mathrm{~s}$, but during the majority of the time the jet may carry heavy baryon loading and is mildly relativistic or non-relativistic so that no bright $\gamma$-rays could be produced during this period of time. One possibility is that the engine is a new-born magnetar which initially injects baryon-loaded, neutrino-driven wind ${ }^{28}$. The advantage of this model is that the $\mathrm{X}$-ray plateau observed in this burst (Methods) may be interpreted as the spindown of such a new-born magnetar. A short duration for this scenario may be achieved by invoking differential-rotation-induced magnetic bubbles as the mechanism of producing GRB prompt emission ${ }^{29}$. With a relatively high initial seed magnetic field strength $B \sim B_{14} \times 10^{14} \mathrm{G}$, the total duration may be estimated as $t \sim 1 B_{14}^{-1} \Omega_{4}^{-1} \mathrm{~s}$, where $B_{14}=B / 10^{14} \mathrm{G}$ and $\Omega_{4}$ is the characteristic differential angular rotation speed in units of $10^{4} \mathrm{~s}^{-1}$ (ref. ${ }^{24}$ ). 
Table 1: Properties of GRB 200826A

\begin{tabular}{|c|c|}
\hline "Duration $\left[T_{90}\right](10-800 \mathrm{keV})$ & $0.96_{-0.08}^{+0.05} \mathrm{~s}$ \\
\hline$f$-parameters $[f]$ & $7.58 \pm 1.23$ \\
\hline Hardness Ratio (50-300/10-50 keV) & 0.803 \\
\hline Spectral photon index $[\alpha]$ & $-0.68 \pm 0.05$ \\
\hline Spectral peak energy $\left[E_{\mathrm{p}}\right]$ & $120.29_{-3.67}^{+3.93} \mathrm{keV}$ \\
\hline Isotropic energy $\left[E_{\gamma, \text { iso }}\right]$ & $7.09 \pm 0.28 \times 10^{51} \mathrm{erg}$ \\
\hline Total fluence & $4.85 \pm 0.19 \times 10^{-6} \mathrm{erg} \mathrm{cm}^{-2}$ \\
\hline$\epsilon$ - parameter & 0.33 \\
\hline Time lag(10-20 keV $\sim 250-300 \mathrm{keV})$ & $0.157 \mathrm{~s}$ \\
\hline Redshift $[z]$ & $0.7481 \pm 0.0003$ \\
\hline Offset $\left[R_{\text {off }}\right]$ & $2.6 \mathrm{kpc}$ \\
\hline Half light radius $\left[R_{50}\right]$ & $5.8 \mathrm{kpc}$ \\
\hline Normalized offset $\left[R_{\text {off }} / R_{50}\right]$ & 0.45 \\
\hline Cumulatve light fraction $\left[F_{\text {light }}\right]$ & 0.79 \\
\hline $\mathcal{O}$ calculated with(out) $f$ & $-3.1(1.5)$ \\
\hline $\log O(\mathrm{II}: \mathrm{I})_{\text {prompt }}$ calculated with(out) $f$ & $-5.2(-0.57)$ \\
\hline $\log O(\mathrm{II}: \mathrm{I})_{\text {host }}$ & 2.5 \\
\hline Peak flux $\left[F_{\mathrm{p}}\right]$ & $9.11_{-1.17}^{+1.47} \times 10^{-6} \mathrm{erg} \mathrm{cm}^{-2} \mathrm{~s}^{-1}$ \\
\hline Peak luminosity $\left[L_{\gamma, \mathrm{p}, \text { iso }}\right]$ & $1.41_{-0.21}^{+0.23} \times 10^{52} \mathrm{erg} \mathrm{s}^{-1}$ \\
\hline Star formation rate $[\mathrm{SFR}]$ & $>1.44 \mathrm{M}_{\odot} \mathrm{yr}^{-1}$ \\
\hline Stellar mass $\left[M_{*}\right]$ & $4.1 \pm 2.9 \times 10^{9} \mathrm{M}_{\odot}$. \\
\hline Specific star formation rate [sSFR] & $>0.35 \mathrm{Gyr}^{-1}$ \\
\hline Optical transient location & $00 \mathrm{~h} 27 \mathrm{~m} 08.5 \mathrm{~s} \quad 34 \mathrm{~d} 01 \mathrm{~m} 38.3 \mathrm{~s}$ \\
\hline $\mathrm{SN}$-association & likely \\
\hline
\end{tabular}



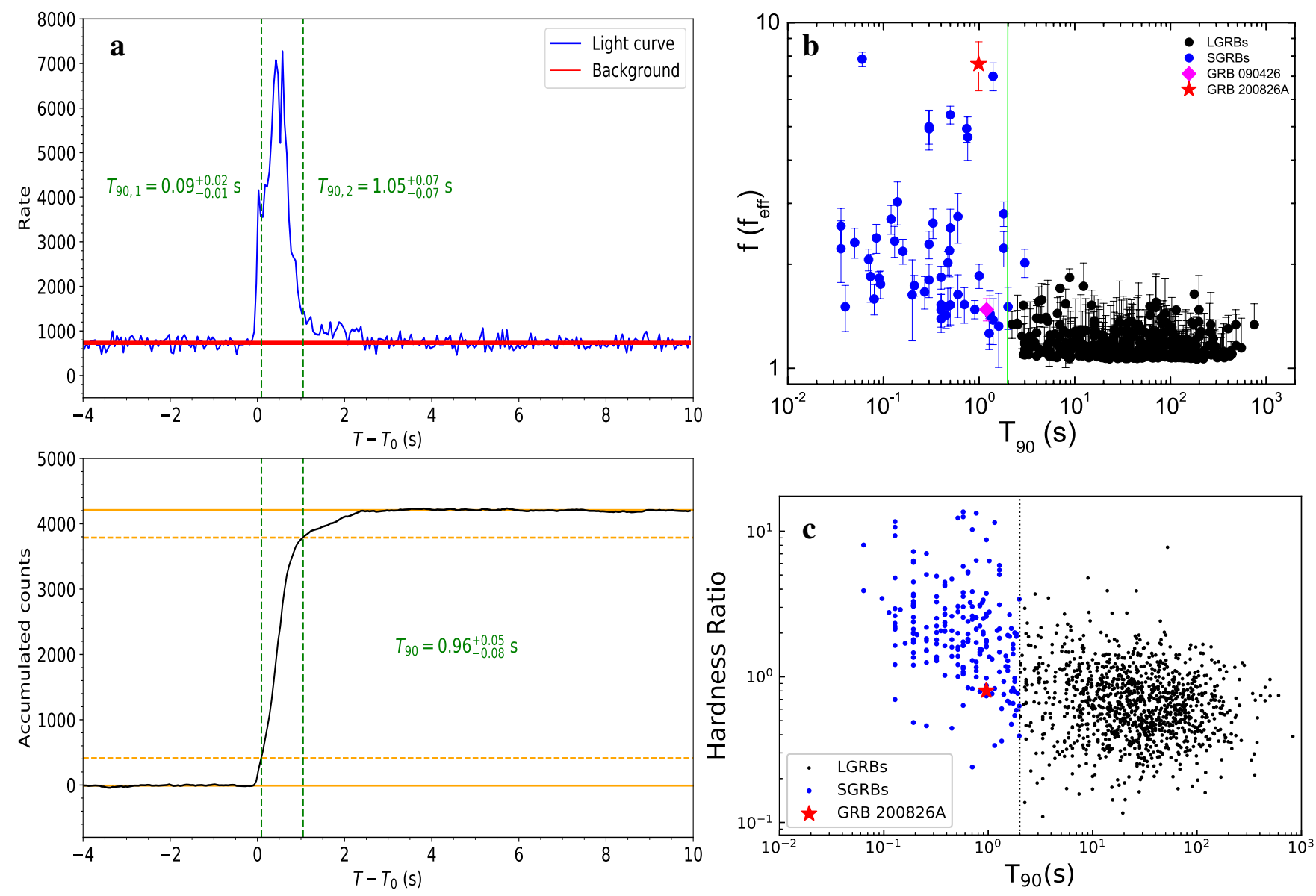

Figure 1: Temporal properties of GRB 200826A. a, $T_{90}$ calculation: The upper panel shows the light curve and background flux level with blue and red solid lines, respectively. The accumulated counts as a function of time are shown with the black curve in the lower panel. The orange horizontal dashed (solid) lines are drawn at 5\%(0\%) and 95\% (100\%) of the total accumulated counts. The $T_{90}$ interval is marked by the green vertical dashed lines. $\mathbf{b}, T_{90}-f\left(f_{\text {eff }}\right)$ plot, where $f_{\text {eff }}$ is the effective $f$ parameter when a long GRB becomes a disguised short GRB by arbitrarily lowering its flux level. GRB 200826A is highlighted by the red solid star. Blue circles correspond to $f$ parameter values of short GRBs, black circles mark $f_{\text {eff }}$ values of long GRBs and green vertical line is the division line at $2 \mathrm{~s}$. c, GRB 200826A on the $T_{90}$-HR diagram : Blue and black points represent the short and long GRBs, respectively; red solid star marks GRB 200826A; and the black dashed line separate long and short GRBs at $2 \mathrm{~s}$. 

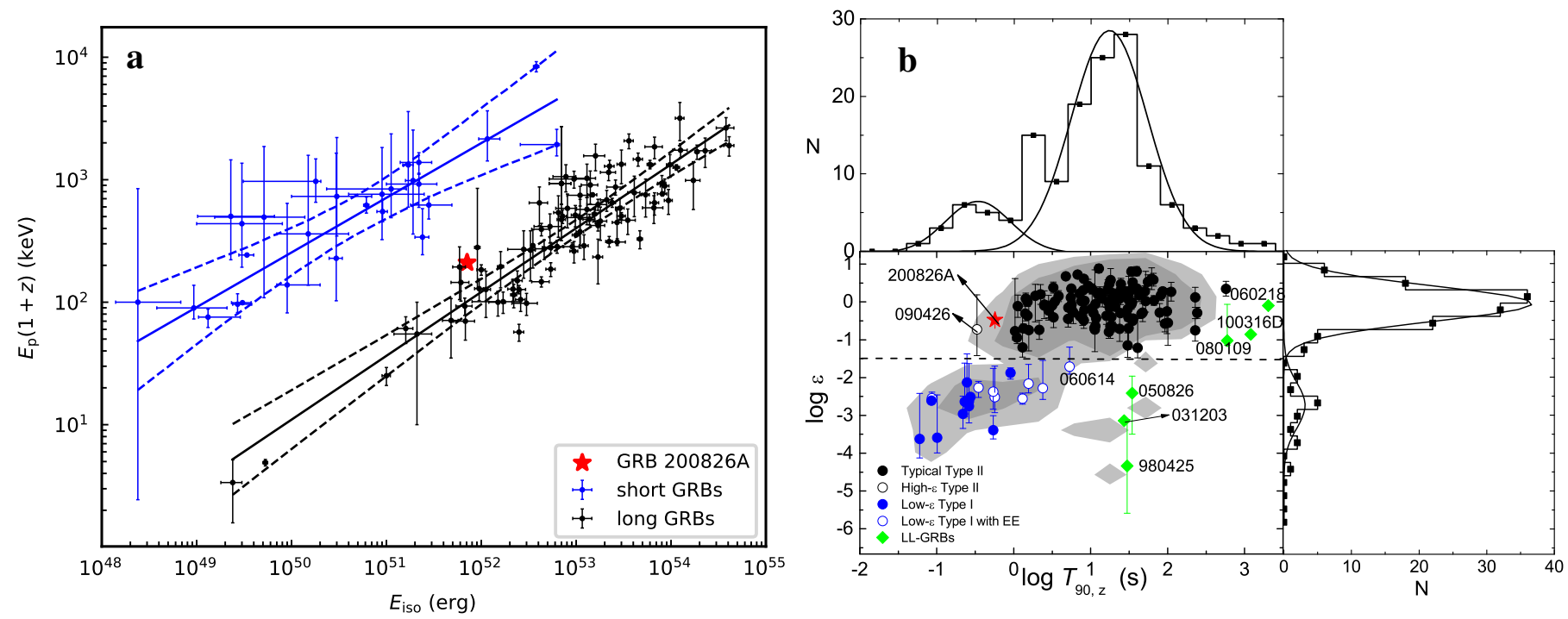

Figure 2: GRB 200826A in energy-related correlations. a, $E_{\mathrm{p}, \mathrm{z}}-E_{\gamma, \text { iso }}$ correlation diagram for short and long GRBs. Dashed borderlines show the $3 \sigma$ regions for each correlation. b, One and two dimensional distributions of GRB samples in the $\epsilon-T_{90}$ space. Blue and black solid circles represent the Type I and Type II GRB candidates, respectively. The special case of the shortduration GRB 090426 with a high- $\epsilon$ is marked by an open black circle. Green diamonds denote the nearby low-luminosity long GRBs. Probability contours of Type I and II GRB clusters are also displayed in the grey regions (see Methods). The dashed horizontal line is the $\epsilon=0.03$ division line. All error bars represent 1- $\sigma$ uncertainties. One can see that GRB 200826A comfortably falls in the Type II region. 

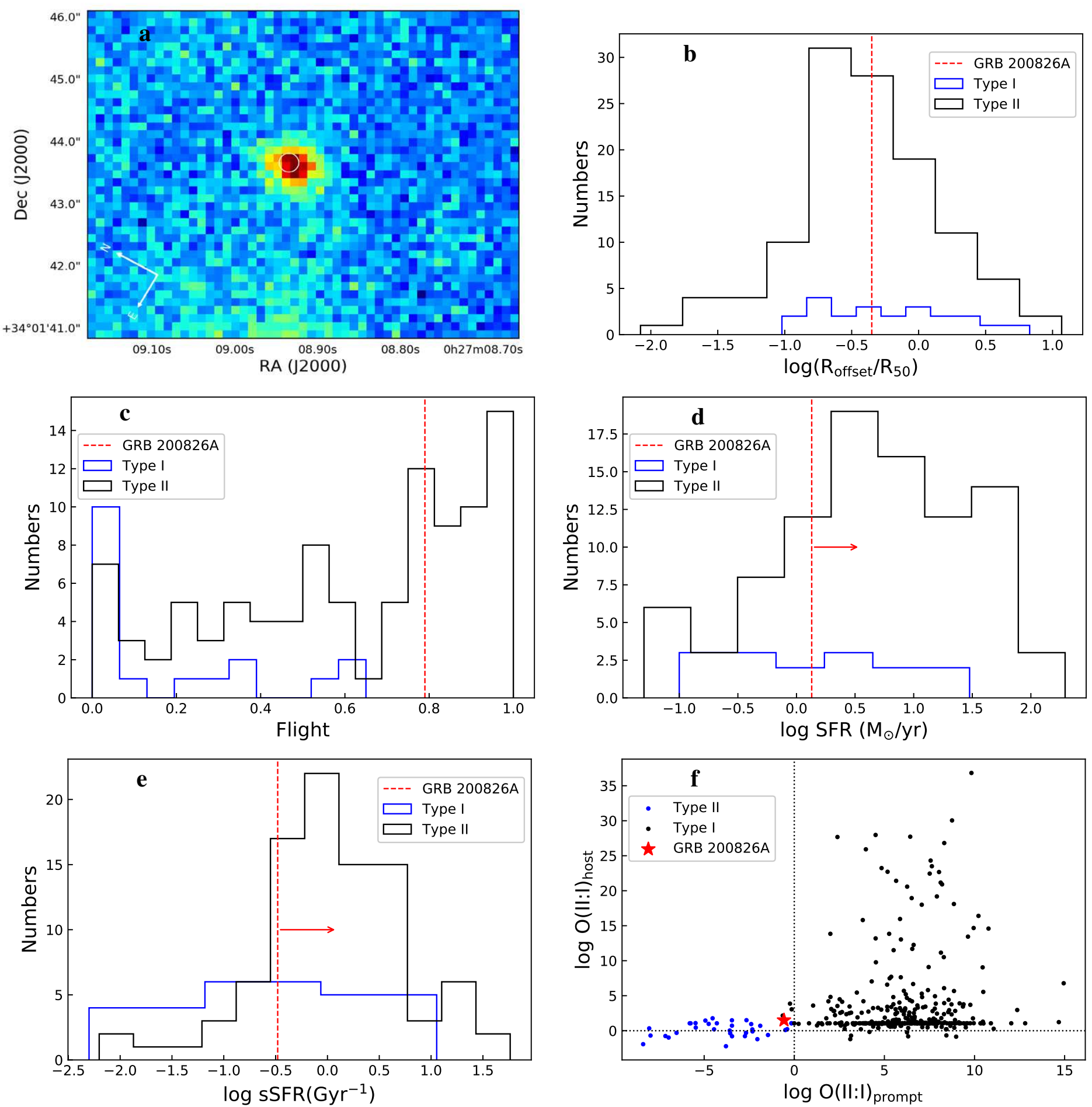

Figure 3: Host properties of GRB 200826A. a, The Gran Telescopio CANARIAS (GTC) Image of GRB 200826A's host galaxy. b, Normalized offset $\left(r=R_{\text {off }} / R_{50}\right)$ distributions of our samples. c, Distributions of cumulative light fraction $F_{\text {light }}$. d, Star formation rate (SFR) of the host galaxies. e, specific star formation rate (sSFR; the ratio between SFR and stellar mass of host galaxy M) of the host galaxies. f, Posterior odds O (II:I) of prompt emission and host properties. GRB 200826A is marked with a red star. In b, $\mathbf{c}, \mathbf{d}$, and e, the black and blue histograms are for Type I and Type II GRBs, respectively. The vertical dashed red lines represent the location of GRB 200826A. 
1. Kouveliotou, C. et al. Identification of Two Classes of Gamma-Ray Bursts. Astrophys. J. 413, L101 (1993).

2. Woosley, S. E. \& Bloom, J. S. The Supernova Gamma-Ray Burst Connection. Ann. Rev. Astron. Astrophys. 44, 507-556 (2006).

3. Abbott, B. P. et al. Multi-messenger Observations of a Binary Neutron Star Merger. Astrophys. J. 848, L12 (2017).

4. Zhang, B. et al. Discerning the Physical Origins of Cosmological Gamma-ray Bursts Based on Multiple Observational Criteria: The Cases of $\mathrm{z}=6.7$ GRB 080913, $\mathrm{z}=8.2$ GRB 090423, and Some Short/Hard GRBs. Astrophys. J. 703, 1696-1724 (2009).

5. Gehrels, N. et al. A new $\gamma$-ray burst classification scheme from GRB060614. Nature 444, 1044-1046 (2006).

6. Levesque, E. M. et al. GRB090426: the environment of a rest-frame 0.35-s gamma-ray burst at a redshift of 2.609. Mon. Not. R. Astron. Soc. 401, 963-972 (2010).

7. Meegan, C. et al. The Fermi Gamma-ray Burst Monitor. Astrophys. J. 702, 791-804 (2009).

8. Sagues Carracedo, A., Dunwoody, R., Meegan, C. \& Fermi GBM Team. GRB 200826A: Fermi GBM observation. GRB Coordinates Network 28287, 1 (2020). URL https:// gen.qstc.nasa.qov/qen3/2828\%.9en3.

9. Ahumada, T. et al. GRB200826A: Zwicky Transient Facility Identifies Optical Afterglow Candidate of a Fermi Short GRB (Trigger 620108997). GRB Coordinates Network 28295, 1 (2020). URL https://acn. asfc.nasa.qov/qcn3/28295. acn3.

10. Rothberg, B., Kuhn, O., Veillet, C. \& Allanson, S. GRB200826A. GRB Coordinates Network 28319, 1 (2020). URL https:// acn. asfc.nasa.qov/acn3/28319. qen3.

11. Ahumada, T., et al. Discovery and confirmation of the shortest Gamma Ray Burst with a collapsar. Nature submitted , (2020).

12. Lü, H.-J., Zhang, B., Liang, E.-W., Zhang, B.-B. \& Sakamoto, T. The 'amplitude' parameter of gamma-ray bursts and its implications for GRB classification. Mon. Not. R. Astron. Soc. 442, 1922-1929 (2014).

13. Amati, L. et al. Intrinsic spectra and energetics of BeppoSAX Gamma-Ray Bursts with known redshifts. Astron. Astrophys. 390, 81-89 (2002). 
14. Lü, H.-J., Liang, E.-W., Zhang, B.-B. \& Zhang, B. A New Classification Method for Gammaray Bursts. Astrophys. J. 725, 1965-1970 (2010).

15. Zhang, B. A burst of new ideas. Nature 444, 1010-1011 (2006).

16. Norris, J. P. et al. Attributes of Pulses in Long Bright Gamma-Ray Bursts. Astrophys. J. 459, 393 (1996).

17. Yi,T.-F., Liang, E.-W., Qin, Y.-P. \& Lu, R.-J. On the spectral lags of the short gamma-ray bursts. Mon. Not. R. Astron. Soc. 367, 1751-1756 (2006).

18. Fruchter, A. S. et al. Long $\gamma$-ray bursts and core-collapse supernovae have different environments. Nature 441, 463-468 (2006).

19. Berger, E. Short-Duration Gamma-Ray Bursts. Ann. Rev. Astron. Astrophys. 52, 43-105 (2014).

20. Li, Y., Zhang, B. \& Yuan, Q. A Comparative Study of Long and Short GRBs. II. A Multiwavelength Method to Distinguish Type II (Massive Star) and Type I (Compact Star) GRBs. Astrophys. J. 897, 154 (2020).

21. Gehrels, N. et al. A short $\gamma$-ray burst apparently associated with an elliptical galaxy at redshift $\mathrm{z}=0.225$. Nature 437, 851-854 (2005).

22. Li, Y., Zhang, B, Lü, H.-J., A Comparative Study of Long and Short GRBs. I. Overlapping Properties. Astrophys. J. 227, 7-38(2016).

23. Zhang, B. Physics of Gamma-ray Burst,418-443 Cambridge University Press, (2018).

24. Belczynski, K., Bulik, T. \& Rudak, B. Study of Gamma-Ray Burst Binary Progenitors. Astrophys. J. 571, 394-412 (2002).

25. Middleditch, J. A White Dwarf Merger Paradigm for Supernovae and Gamma-Ray Bursts. Astrophys. J. 601, L167-L170 (2004).

26. Vietri, M.\& Stella, L. A Gamma-Ray Burst Model with Small Baryon Contamination. Astrophys. J. 507, 2031-2056 (2011).

27. Bromberg, O., Nakar, E., Piran, T., Are Low-luminosity Gamma-Ray Bursts Generated by Relativistic Jets?, Astrophys. J. 739, L55-L59(2011). 
28. Metzger, N. et al. The protomagnetar model for gamma-ray bursts. Mon. Not. R. Astron. Soc. 413, L45-L48 (1998).

29. Kluźniak, W. and Ruderman, M.. The Central Engine of Gamma-Ray Bursters. Astrophys. J. 505, L113-L117 (1998).

Acknowledgements B.B.Z acknowledges support by the National Key Research and Development Programs of China (2018YFA0404204), the National Natural Science Foundation of China (grant Nos. 11833003, U2038105), and the Program for Innovative Talents, Entrepreneur in Jiangsu. We also acknowledge the use of public data from the Fermi Science Support Center (FSSC).

Author Contributions BBZ and HJL initiated the study. BBZ and BZ coordinated the scientific investigations of the event. BBZ, ZKL, ZKP, YL, HJL, JY, YSY, YHY, YZM and JHZ processed and analyzed the data. AJCT and YDH carried out the GTC optical observations. JRM, XHZ and JMB carried out the Lijiang $2.4 \mathrm{~m}$ optical observations. XGW carried out the LCOGT observations. BZ and ZGD contributed to the theoretical interpretations to the event. BBZ and BZ wrote the paper with the contributions from all coauthors.

Competing Interests The authors declare that they have no competing financial interests.

Correspondence Correspondence and requests for materials should be addressed to B.-B. Zhang (bbzhang@nju.edu.cn) and B. Zhang (zhang@physics.unlv.edu)

Data Availability Statement Processed data are presented in the tables and figures in the paper. Source data are available upon reasonable requests to the corresponding authors. The Fermi/GBM data are publicly available at https://heasarc.qsfc.nasa.qov/FTP/fermi/data/. 


\section{Methods}

Fermi data analysis. We extracted photon events collected by the Sodium Iodide (NaI) scintillation detector $\mathrm{nb}$ whose boresight has the smallest angle relative to the burst location and conduct the following temporal and spectral analysis as described in Ref. $\frac{\text { [0 }}{}$.

Light curve analysis: We subtracted the background from the light curves using the approach described in Ref. ${ }^{31}$. From the sharp peak from $T_{0}+0.09$ s to $T_{0}+1.05 \mathrm{~s}$ (Figure Ma), we obtained its $T_{90} \sim 0.96 \mathrm{~s}$, representing the time between the epochs when $5 \%$ and $95 \%$ of the total fluence are collected.

Spectral analysis: We conducted both the time-integrated and time-resolved spectral analysis during the $T_{90}$ time interval. The time-dependent spectral analysis is performed by slicing $T_{90}$ into 19 segments with a bin size of 0.05 seconds. Each segment is guaranteed to have at least 20 net photon counts. The background spectra were obtained by modeling the data in the signal-free time interval using the baseline method ${ }^{[31}$. We used GBM Response Generator to generate the detector response matrices (DRMs). Finally, we adopted $M c S p e c F i t^{311}$ to fit both preprocessed spectra and plotted the best fitting results. The mainstream spectra fitting models, such as the single power law (PL), cutoff power law (CPL), band function (Band), black body (BB), and their hybrids were utilized to fit both spectra. By assessing the fitting performance via the combination of Profile-Gaussian likelihood (PGSTAT/dof) and Bayesian information criterion $(\mathrm{BIC})^{321}$, the CPL model was favored for best representing the properties of both time-integrated and time-resolved spectra. The CPL model is described in the form of

$$
N(E)=A\left(\frac{E}{100 \mathrm{keV}}\right)^{\alpha} \exp \left[\frac{-(\alpha+2) \mathrm{E}}{\mathrm{E}_{\mathrm{p}}}\right]
$$

where $\alpha$ is the photon spectral index, $A$ is the normalized coefficient, and $E_{\mathrm{p}}$ is the peak energy of the spectrum. We fitted time-dependent spectra in 19 aforementioned time intervals using the CPL model. Fitting results demonstrate an intensity tracking pattern of spectral evolution, which is shown in Extended Data Figure W. Time-integrated fitting results are exhibited in Extended Data Figure 2. We list the results in Extended Data Table 1].

Amplitude parameter. The $f$ parameter is defined by $f \equiv \frac{F_{\mathrm{p}}}{F_{\mathrm{b}}}$, where $F_{\mathrm{p}}$ and $F_{\mathrm{b}}$ correspond to the peak flux and average background flux of a GRB, respectively. In order to distinguish intrinsically short GRBs from disguised short GRBs due to the tip-of-iceberg effect, an effective amplitude parameter $f_{\text {eff }}=\frac{F_{\mathrm{p}^{\prime}}}{F_{\mathrm{b}}}$ for long GRBs is defined, which is the $f$ value of "pseudo GRBs" whose flux is re-scaled from the initial flux so that $T_{90}$ above the background flux is

\footnotetext{
iihttps://fermi.asfc.nasa.qov/ssc/data/analysis/rmfit/abmrsp-2.0.10.tar.bz2
} 
shorter than $2 \mathrm{~s}$ [2] By comparing the $f-T_{90}$ diagram of short GRBs and the $f_{\text {eff }}-T_{90}$ diagram of long GRBs, one can see that most of intrinsically short GRBs possess relatively large $f$ than $f_{\text {eff }}$ of long GRBs. A disguised short GRB would have $f<1$.5. GRB 200826A has $f=7.58 \pm 1.23$, which is much greater than the range for a disguised GRB. As a result, this GRB is a genuinely short GRB.

Hardness ratio. Long GRBs are usually softer than short GRBs, suggesting that they have a lower hardness ratio (HR). We define HR as the photon counts ratio between 50-300 keV and 10-50 keV energy bands. The GRB sample (from Ref ${ }^{[33}$ ) and GRB 200826A are marked in Figure $\Pi c$ by points and star, respectively.

Amati relation. The correlation between the GRB isotropic energy $E_{\gamma \text {,iso }}$ and the rest-frame peak energy $E_{\mathrm{p}, \mathrm{z}}=(1+z) E_{\mathrm{p}}$ was discovered by Amati et al. ${ }^{[34}$, and can be written as $\log E_{\mathrm{p}, \mathrm{z}}=a+b \log E_{\gamma, \text { iso }}$. Typically, long and short GRBs follow different tracks in the $E_{\mathrm{p}, \mathrm{z}}-E_{\gamma, \text { iso }}$ diagram. We over-plotted the $E_{\mathrm{p}, \mathrm{z}}-E_{\gamma, \text { iso }}$ diagram (Figure [la) ${ }^{\text {mil }}$ using GRB samples with known redshift from Refs ${ }^{4}$ and ${ }^{34}$. Utilising the MCMC method, the optimal fitting parameters and $3 \sigma$ uncertainties are constrained. We obtain $a=-25.01_{-4.25}^{+3.91}$ and $b=0.52_{-0.07}^{+0.08}$ for long GRBs, and $a=-19.92_{-7.60}^{+9.18}$ and $b=0.45_{-0.18}^{+0.15}$ for short GRBs.

$\epsilon$ parameter. With the parameter $\epsilon=E_{\gamma, \text { iso }, 52} / E_{\mathrm{p}, \mathrm{z}, 2}^{5 / 3}$, where $E_{\gamma, \text { iso }, 52}=E_{\gamma, \text { iso }} / 10^{52} \mathrm{erg}$ and $E_{\mathrm{p}, \mathrm{z}, 2}=E_{\mathrm{p}, \mathrm{z}} / 100 \mathrm{keV}$, a classification method ${ }^{\text {144 }}$ was proposed to clearly divide GRBs with high $\epsilon(\epsilon>0.03)$ and low $\epsilon(\epsilon<0.03)$ at the separation line $\epsilon \sim 0.03$. The two classes nicely match Type II and Type I GRBs, respectively. We computed $\epsilon$ of GRB 200826A and plotted it on the $\log T_{90, z}-\log \epsilon$ panel (Figure $2 \mathrm{~b}$ ). The classification of a GRB can be quantitatively evaluated by a GRB distribution probability, which is defined as the number of GRBs falling inside an area of $d \log \epsilon$ by $d \log T_{90, z}$ centered at $\log \epsilon, \log T_{90, z}$ divided by the total number of the GRB samples. We assume this probability of each GRB in our sample $p_{i}$ has a normalized $\log$-normal probability distribution $p(\log \epsilon, \delta \log \epsilon)$. We calculated the integral of $p_{i}$ over the range $[\log \epsilon-d \log \epsilon / 2, \log \epsilon+d \log \epsilon / 2]$ and summed over the entire GRB sample to obtain the probability $P\left(\log \epsilon, \log T_{90, \mathrm{z}}\right)$. Dark grey $\left(P\left(\log \epsilon, \log T_{90, \mathrm{z}}\right)>0.1\right)$ and light grey $\left(P\left(\log \epsilon, \log T_{90, z}\right)>0.003\right.$ at $3 \sigma$ confidence level) regions in Figure $2 \mathrm{~b}$ mark probability contours of GRB clustering, respectively. GRB 200826A falls into the high $\epsilon$ region with $P\left(\log \epsilon, \log T_{90, z}\right)>0.003$, which suggests that it is of a Type II origin.

Spectral lag. For most GRBs, time lags among different bands, also known as spectral lags, exist in the light curves ${ }^{[5]}$ due to the fact that photons with different energies arrive the observer at different times. We used the cross-correlation function $(\mathrm{CCF})^{[6,5]}$ to calculate the time lags

\footnotetext{
${ }^{\text {iii }} \mathrm{A}$ cosmology with $H 0=67.7 \mathrm{~km} \mathrm{~s}^{-1} \mathrm{Mpc}^{-1}, \Omega_{\mathrm{m}}=0.307$ and $\Omega_{\Lambda}=0.693$ is applied in this paper.
} 
of light curves among different energy bands between $T_{0}+0.5 \mathrm{~s}$ and $T_{0}+1.6 \mathrm{~s}$ following

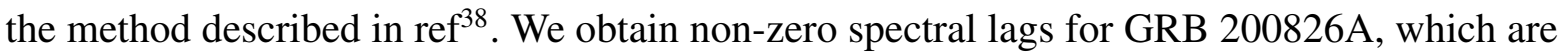
shown in Extended Data Figure [3].

X-ray Observations. The Swift/XRT has performed a follow-up observation for a duration

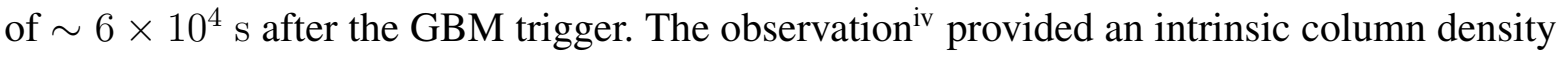
of $6.0 \times 10^{20} \mathrm{~cm}^{-2}$. The X-ray light curve is shown in the Extended Data Figure 4. We fitted the light curve using a broken power-law model and obtained the decay slopes as $\sim-1.5$ and $\sim-0.4$, and a break at $\sim 10^{5} \mathrm{~s}$.

Optical transient. An optical counterpart of GRB 200826A was located by Zwicky Transient Facility ${ }^{\underline{g}}(\mathrm{ZTF})$ and followed up by several ground and space facilities. We summarized our own observations performed by the Gran Telescopio CANARIAS (GTC), Lijiang 2.4-meter telescope, and LCOGT network observatory in Extended Data Table 2. Other sets of optical observations can be found in Ref.

Host galaxy observations and properties. We observed the host galaxy with the Gran Telescopio CANARIAS (GTC) in $r$-band, LCOGT network observatory in $i$-band. Those results allow us to perform the following analyses on the host galaxy properties.

- $R_{50}$. The ZTF position is used to estimate the half-light radius $R_{50}$. We used the PyRAF/ellipse routine to generate elliptical intensity isophotes, constructed the total flux profile as a function of semi-major axis, and calculated the half-light radius, $R_{50}$, through interpolation. We obtained $R_{50} \sim 0.77$ ", corresponding to $5.7 \mathrm{kpc}$ at redshift 0.7481 . With a $0.35^{\prime \prime}$ offset mentioned by Ahumada, T. et al. ${ }^{\underline{\square}}$, the normalized offset $R_{\text {off }} / R_{50}=0.45$ is a typical value for LGRBs.

- $F_{\text {light }}$. We estimated the cumulative light fraction $F_{\text {light }}$ with our GTC image. In the intensity versus total flux profile, we interpolated the mean intensity at the position of GRB 200826A to estimate the total flux of the fainter regions and $F_{\text {light }}=F_{\text {fainter }} / F_{\text {total }}=$ 0.79. The offsets and $F_{\text {light }}$ of GRB 200826A are typical for LGRBs. Comparatively, GRB 090426A has a smaller offset and larger $F_{\text {light }}$ than GRB 200826A.

- SFR. We calculated the star formation rate (SFR) with near-UV (NUV) observations (and emission lines). Since the rest frame wavelength of the $g$-band is in NUV, we are able to approximate the SFR with the Galactic extinction corrected $g$-band magnitude from DESI, as 8.7 $M_{\odot} \mathrm{yr}^{-1}$ based on Kennicutt $1998^{139}$. The [OII] $\lambda 3727$ emission line flux present in the GTC spectrum ${ }^{\square}$ is $2.81 \times 10^{-17} \mathrm{erg} \mathrm{s}^{-1} \mathrm{~cm}^{-2}$, which corresponds to a

${ }^{i v}$ https://www.swift.ac.uk/xrt_live_cat/00021028/ 
luminosity $1.03 \times 10^{41} \mathrm{erg} \mathrm{s}^{-1}$. The SFR estimated with Ref. ${ }^{[39}$ using the [OII] emission line is $(1.4 \pm 0.4) \times 10^{-41} L_{[\mathrm{OII}]}>\mathbf{1 . 4 4} M_{\odot} \mathrm{yr}^{-1}$. Emission lines represent star formation rate with age $0-10 \mathrm{Myr}$, which is more related to the origin of LGRBs, hence serves as a better indicator of distinguishing LGRBs and SGRBs. We thus used the SFR estimated with [OII] emission line to obtain the Odds O(II:I) (see below).

- Stellar mass. To estimate the stellar mass, we used the Code Investigating GALaxy Emission (CIGALE) 40 - to perform Spectral Energy Distribution (SED) fitting in $g, r, i$ and $z$ bands after considering the Galactic extinction ${ }^{41}$. Several models, including the sfhdelayed star formation history model with an initial SFR of $0.1 M_{\odot} \mathrm{yr}^{-1}, b c 03$ stellar population model ${ }^{42}$ with a Chabrier initial mass function, dustatt_calzleit dust attenuation model with the UV bump centroid to be $217.5 \mathrm{~nm}$, dale 2014 dust emission model ${ }^{\sqrt{43}}$ with various AGN fractions, were taken into account. Our results yield a stellar mass of $M_{*}=(4.1 \pm 2.9) \times 10^{9} M_{\odot}$. Correspondingly, the specific star formation rate (sSFR) can be calculated as $>0.35 \mathrm{Gyr}^{-1}$.

$\mathcal{O}$. A naive Bayesian scheme has been proposed by Li et al., $2020^{20}$ to determine the physical categories of GRBs based on multi-wavelength properties. The parameter $\mathcal{O}=\log \mathrm{O}(\mathrm{II}: \mathrm{I})+$ 0.7 is the logarithmic Type II to Type I probability ratio corrected for the potential correlation between parameters. We calculate $\mathcal{O}$ based on the parameter sets from the prompt emission and host galaxy properties. A GRB is classified as a Type II or Type I if $\mathcal{O}$ is positive or negative. Without inclusion of the $f$ parameter, we obtain a positive value $\mathcal{O}=1.5$, placing GRB 200826A in the Type II rather than Type I GRB category. With the method proposed in ref. ${ }^{[20}$, we further separated the parameter sets into groups of prompt emission and host galaxy to compute $\log \mathrm{O}(\mathrm{II}: \mathrm{I})_{\text {prompt }}$ and $\log \mathrm{O}(\mathrm{II}: \mathrm{I})_{\text {host }}$, respectively, and place GRB 200826A on the distribution diagram (Figure Bff).

\footnotetext{
${ }^{\mathrm{v}}$ cigale.oamp.fr
} 
30. Zhang, B.-B. et al. A Comprehensive Analysis of Fermi Gamma-ray Burst Data. I. Spectral Components and the Possible Physical Origins of LAT/GBM GRBs. Astrophys. J. 730, 141 (2011).

31. Zhang, B. B. et al. Transition from fireball to Poynting-flux-dominated outflow in the threeepisode GRB 160625B. Nature Astronomy 2, 69-75 (2018).

32. Zhang, B. B. et al. A peculiar low-luminosity short gamma-ray burst from a double neutron star merger progenitor. Nature Communications 9, 447 (2018).

33. Goldstein,A. et al. An Ordinary Short Gamma-Ray Burst with Extraordinary Implications:Fermi-GBM Detection of GRB 170817A. Astrophys. J. 848, L14 (2017).

34. Amati, L. et al. Intrinsic spectra and energetics of BeppoSAX Gamma-Ray Bursts with known redshifts. Astron. Astrophys. 390, 81-89 (2002).

35. Wei, J.-J., Zhang, B.-B., Shao, L., Wu, X.-F. \& Mészáros, P. A New Test of Lorentz Invariance Violation: The Spectral Lag Transition of GRB 160625B. Astrophys. J. 834, L13 (2017).

36. Norris, J. P., Marani, G. F. \& Bonnell, J. T. Connection between Energy-dependent Lags and Peak Luminosity in Gamma-Ray Bursts. Astrophys. J. 534, 248-257 (2000).

37. Ukwatta, T. N. et al. Spectral Lags and the Lag-Luminosity Relation: An Investigation with Swift BAT Gamma-ray Bursts. Astrophys. J. 711, 1073-1086 (2010).

38. Zhang, B.-B. et al. Unusual Central Engine Activity in the Double Burst GRB 110709B. Astrophys. J. 748, 132 (2012).

39. Kennicutt, J., Robert C. The Global Schmidt Law in Star-forming Galaxies. Astrophys. J. 498, 541-552 (1998).

40. S. Noll, D. Burgarella, E. Giovannoli, V. Buat, D. Marcillac, and J. C. Muñoz-Mateos. Analysis of galaxy spectral energy distributions from far-UV to far-IR with CIGALE: studying a SINGS test sample. Astron. Astrophys. 507, 1793-1813 (2009).

41. Edward F. Schlafly and Douglas P. Finkbeiner. Measuring Reddening with Sloan Digital Sky Survey Stellar Spectra and Recalibrating SFD. Astrophys. J. 737, 103 (2011).

42. G. Bruzual and S. Charlot. Stellar population synthesis at the resolution of 2003. Mon. Not. R. Astron. Soc. 344, 1000-1028 (2003). 
43. Daniel A. Dale, George Helou, Georgios E. Magdis, Lee Armus, Tanio Díaz-Santos, and Yong Shi. A Two-parameter Model for the Infrared/Submillimeter/Radio Spectral Energy Distributions of Galaxies and Active Galactic Nuclei. Astrophys. J. 784, 83 (2004). 


\section{Extended Data}

Extended Data Table W: Time-integrated and time-resolved spectral fitting results of GRB 200826A with cutoff power law model.

Extended Data Table 2]: Observations of optical counterpart and host galaxy of GRB 200826A

Extended Data Figure D: Spectral evolution of GRB 200826A.

Extended Data Figure ․: Spectral fitting results within the interval from $T_{0}+0.50 \mathrm{~s}$ to $T_{0}+1.60 \mathrm{~s}$.

Extended Data Figure 3]: Spectral lag calculation

Extended Data Figure 4: X-ray afterglow of GRB 200826A with broken power-law fitting. 
Extended Data Table 1: Time-integrated and time-resolved spectral fitting results of GRB 200826A with cutoff power law model

\begin{tabular}{|c|c|c|c|c|}
\hline $\mathrm{t} 1-\mathrm{t} 2(\mathrm{~s})$ & $\alpha$ & $E_{\mathrm{p}}(\mathrm{keV})$ & PGSTAT/dof & $\mathrm{BIC}$ \\
\hline $0.50-1.60$ & $-0.68 \pm 0.05$ & $120.29_{-3.67}^{+3.93}$ & $595.5 / 347$ & 613.07 \\
\hline $0.50-0.60$ & $-0.51_{-0.34}^{+0.41}$ & $135.27_{-14.42}^{+35.55}$ & $188.4 / 347$ & 205.97 \\
\hline $0.60-0.65$ & $0.11_{-0.41}^{+0.52}$ & $88.88_{-10.73}^{+14.94}$ & $169.1 / 347$ & 186.68 \\
\hline $0.65-0.70$ & $1.50_{-0.99}^{+0.81}$ & $56.85_{-3.76}^{+8.12}$ & $155.6 / 347$ & 173.19 \\
\hline $0.70-0.75$ & $1.34_{-0.66}^{+0.69}$ & $56.76_{-4.53}^{+4.84}$ & $175.1 / 347$ & 192.65 \\
\hline $0.75-0.80$ & $0.67_{-0.48}^{+0.65}$ & $50.92_{-3.90}^{+4.10}$ & $195.6 / 347$ & 213.13 \\
\hline $0.80-0.85$ & $0.89_{-0.53}^{+0.74}$ & $57.89_{-4.56}^{+6.38}$ & $174.8 / 347$ & 192.37 \\
\hline $0.85-0.90$ & $0.09_{-0.34}^{+0.39}$ & $85.35_{-8.68}^{+10.07}$ & $188.8 / 347$ & 206.34 \\
\hline $0.90-0.95$ & $-0.42_{-0.20}^{+0.16}$ & $137.62_{-10.94}^{+16.87}$ & $209.6 / 347$ & 227.17 \\
\hline $0.95-1.00$ & $-0.62_{-0.17}^{+0.14}$ & $146.88_{-13.52}^{+21.55}$ & $195.1 / 347$ & 212.66 \\
\hline $1.00-1.05$ & $-0.65_{-0.16}^{+0.13}$ & $212.36_{-19.60}^{+39.28}$ & $211.4 / 347$ & 228.96 \\
\hline $1.05-1.10$ & $0.29_{-0.24}^{+0.32}$ & $98.21_{-7.06}^{+6.82}$ & $189.4 / 347$ & 207.01 \\
\hline $1.10-1.15$ & $0.12_{-0.33}^{+0.25}$ & $80.44_{-5.10}^{+7.98}$ & $174.2 / 347$ & 191.80 \\
\hline $1.15-1.20$ & $-0.42_{-0.19}^{+0.16}$ & $159.84_{-14.12}^{+23.83}$ & $226.4 / 347$ & 224.01 \\
\hline $1.20-1.25$ & $0.01_{-0.28}^{+0.26}$ & $90.98_{-6.55}^{+8.09}$ & $158.4 / 347$ & 175.93 \\
\hline $1.25-1.30$ & $0.39_{-0.51}^{+0.47}$ & $67.00_{-4.91}^{+9.52}$ & $177.2 / 347$ & 194.75 \\
\hline $1.30-1.35$ & $0.60_{-0.62}^{+0.66}$ & $49.88_{-4.41}^{+5.97}$ & $130.7 / 347$ & 148.30 \\
\hline $1.35-1.40$ & $0.21_{-0.90}^{+1.17}$ & $40.46_{-5.88}^{+6.16}$ & $129.4 / 347$ & 146.93 \\
\hline $1.40-1.45$ & $-0.17_{-1.42}^{+0.99}$ & $32.51_{-5.19}^{+11.21}$ & $137.0 / 347$ & 154.56 \\
\hline $1.45-1.60$ & $-0.86_{-0.56}^{+0.69}$ & $33.58_{-7.86}^{+5.66}$ & $162.9 / 347$ & 180.45 \\
\hline
\end{tabular}


Extended Data Table 2: Observations of optical counterpart and host galaxy of GRB 200826A

\begin{tabular}{|c|c|c|c|c|c|}
\hline Time(day) & Telescope & Band & System & Magnitude* & Flux Density $(\mu \mathrm{Jy})$ \\
\hline \multicolumn{6}{|c|}{ Optical counterpart } \\
\hline $3.99 \pm 0.002$ & Gran Telescopio Canarias & $\mathrm{r}$ & $\mathrm{AB}$ & $>24.9$ & $<0.40$ \\
\hline $72.05 \pm 0.006$ & LCOGT network observatory & $\mathrm{i}$ & $\mathrm{AB}$ & $>22.7$ & $<3.02$ \\
\hline 72.48 & Lijiang 2.4-meter telescope & $\mathrm{g}$ & $\mathrm{AB}$ & $>22.58$ & $<3.37$ \\
\hline 72.48 & Lijiang 2.4-meter telescope & $\mathrm{r}$ & $\mathrm{AB}$ & $>22.07$ & $<5.40$ \\
\hline 72.48 & Lijiang 2.4-meter telescope & $\mathrm{i}$ & $\mathrm{AB}$ & $>21.81$ & $<6.85$ \\
\hline \multicolumn{6}{|c|}{ Host galaxy } \\
\hline $3.99 \pm 0.002$ & Gran Telescopio Canarias & $\mathrm{r}$ & $\mathrm{AB}$ & $22.45 \pm 0.06$ & $3.80_{-0.18}^{+0.19}$ \\
\hline $72.05 \pm 0.006$ & LCOGT network observatory & $\mathrm{i}$ & $\mathrm{AB}$ & $22.04 \pm 0.39$ & $5.55_{-1.39}^{+2.29}$ \\
\hline
\end{tabular}

* Corrected for foreground Galactic extinction on the line of sight (Ref. ${ }^{411}$ ). 


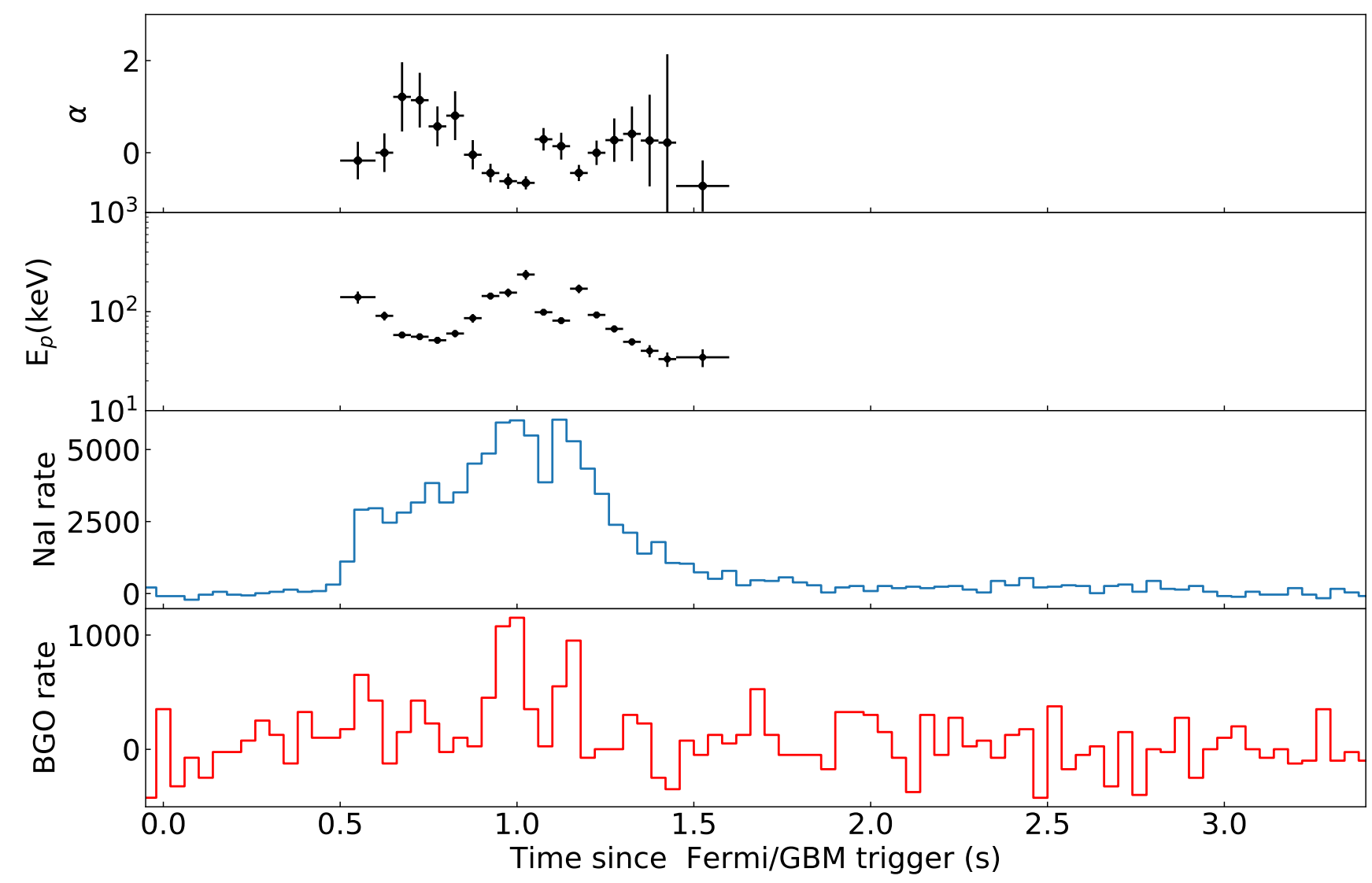

Extended Data Figure 1: Spectral evolution of GRB 200826A. Top two panels: The best-fit parameters of the CPL model in each slices. Lower two panels: The NaI and BGO light curves. All error bars represent $1-\sigma$ uncertainties. 

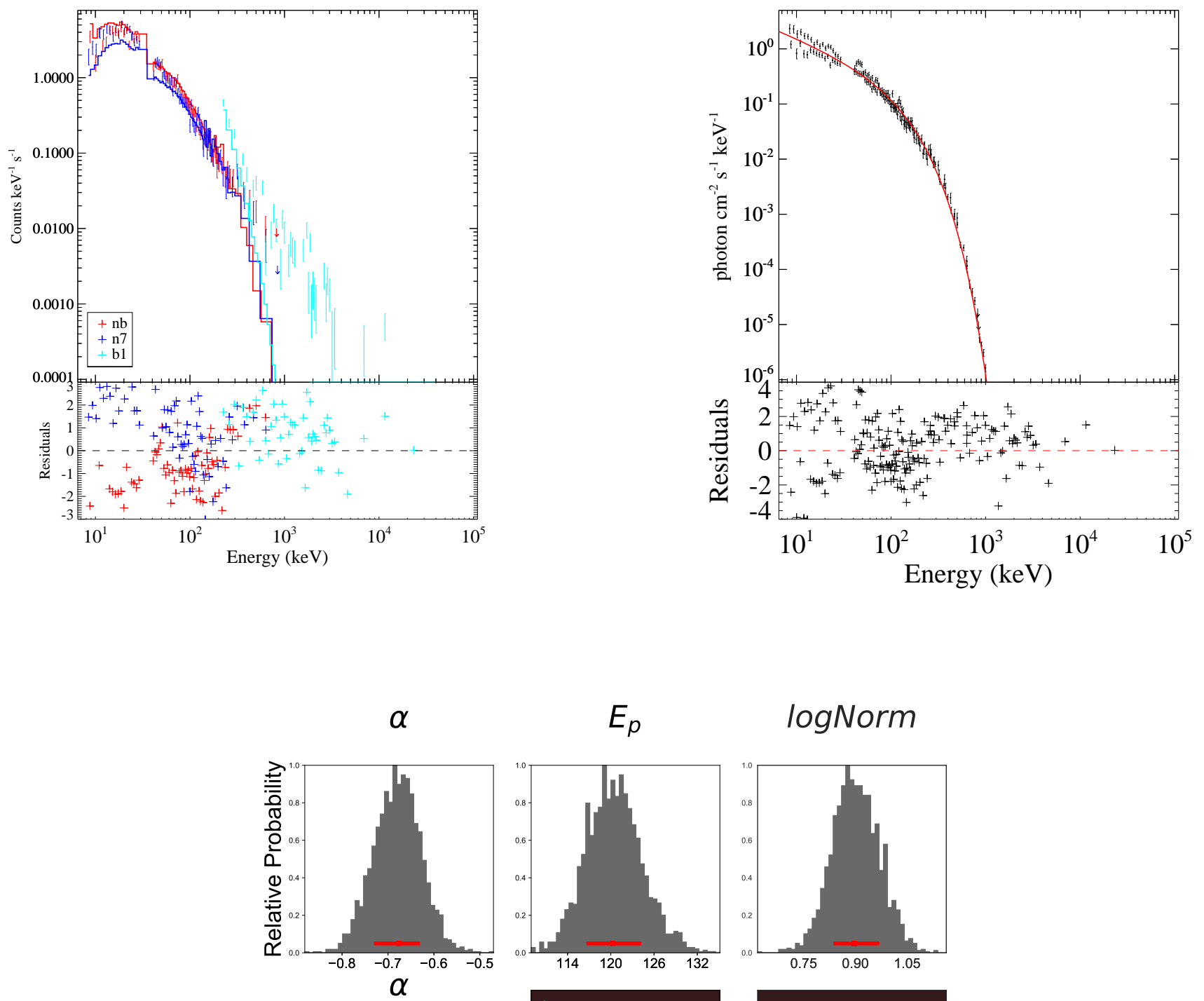

$E_{p}$

$\log$ Norm
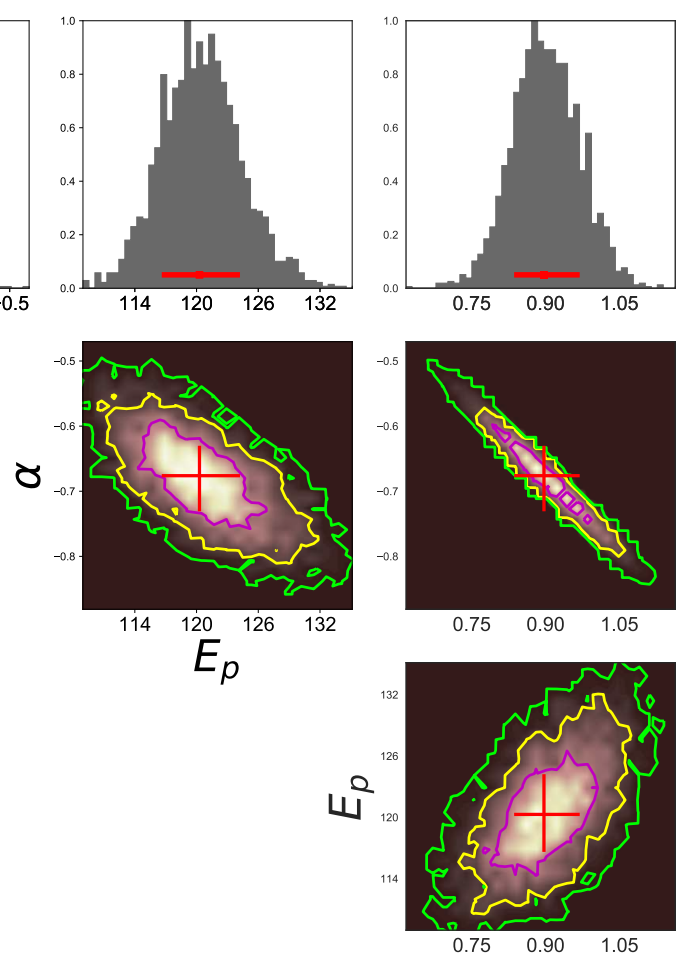

logNorm

Extended Data Figure 2: Spectral fitting results within the interval of $T_{0}+0.50 \mathrm{~s} \sim T_{0}+1.60 \mathrm{~s}$. Top left: photon count spectra overplot with the best-fit model and residuals. Top right: de-convolved spectra and best-fit model. Bottom: corner plot of the cutoff power-law model parameters which shows one and two-dimensional posterior probability distributions of parameters. All error bars represent $1-\sigma$ uncertainties. 


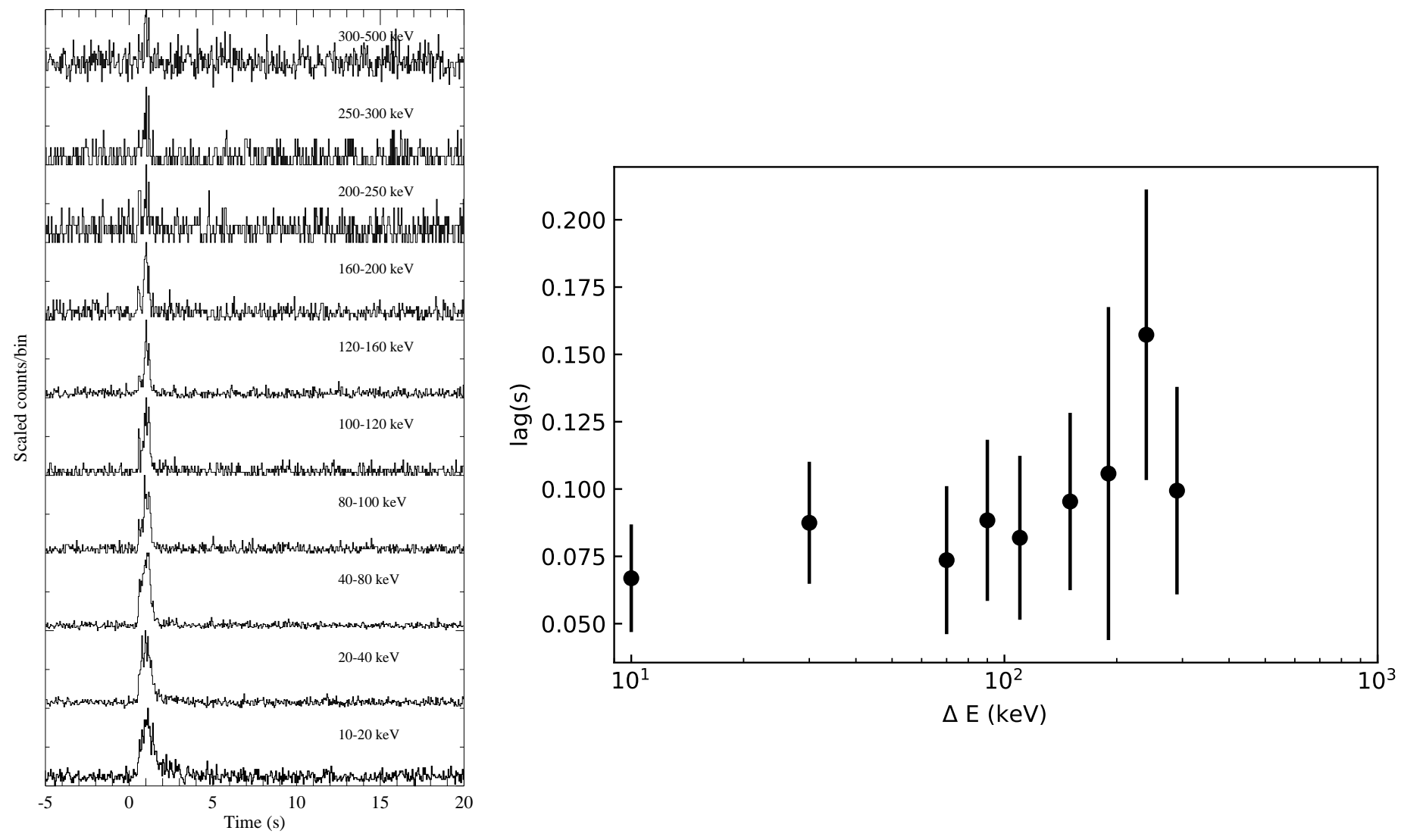

Extended Data Figure 3: Spectral lag calculation. Left: light curves in different energy bands(10$20 \mathrm{keV} \sim 300-500 \mathrm{keV}$ ) which are to calculate lags. Right: energy dependent spectral lag between the lowest energy $(10-20 \mathrm{keV})$ band and any higher energy band. Black points represent any two different energy band time lags and their uncertainties are shown with black bars. All error bars represent $1-\sigma$ uncertainties. 


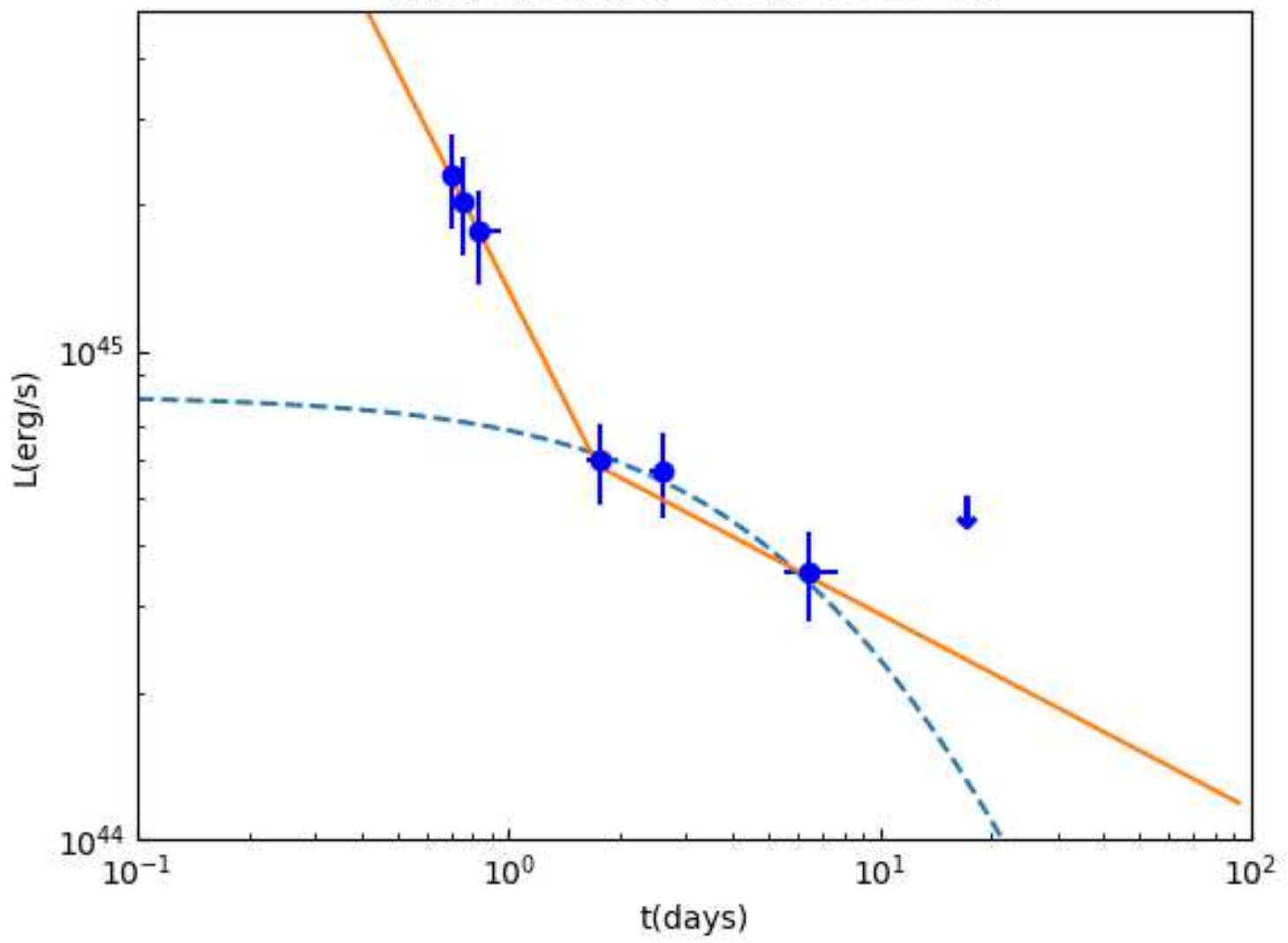

Extended Data Figure 4: X-ray afterglow of GRB 200826A with broken power-law fitting. The slopes are $-1.41_{-0.12}^{+0.24},-0.43_{-0.22}^{+0.17}$ respectively, with a break at $1.51_{-0.30}^{+0.31} \times 10^{5} \mathrm{~s}$. All error bars represent $1-\sigma$ uncertainties. The dashed line is fitted by magnetar spin-down energy injection. 


\section{Figures}
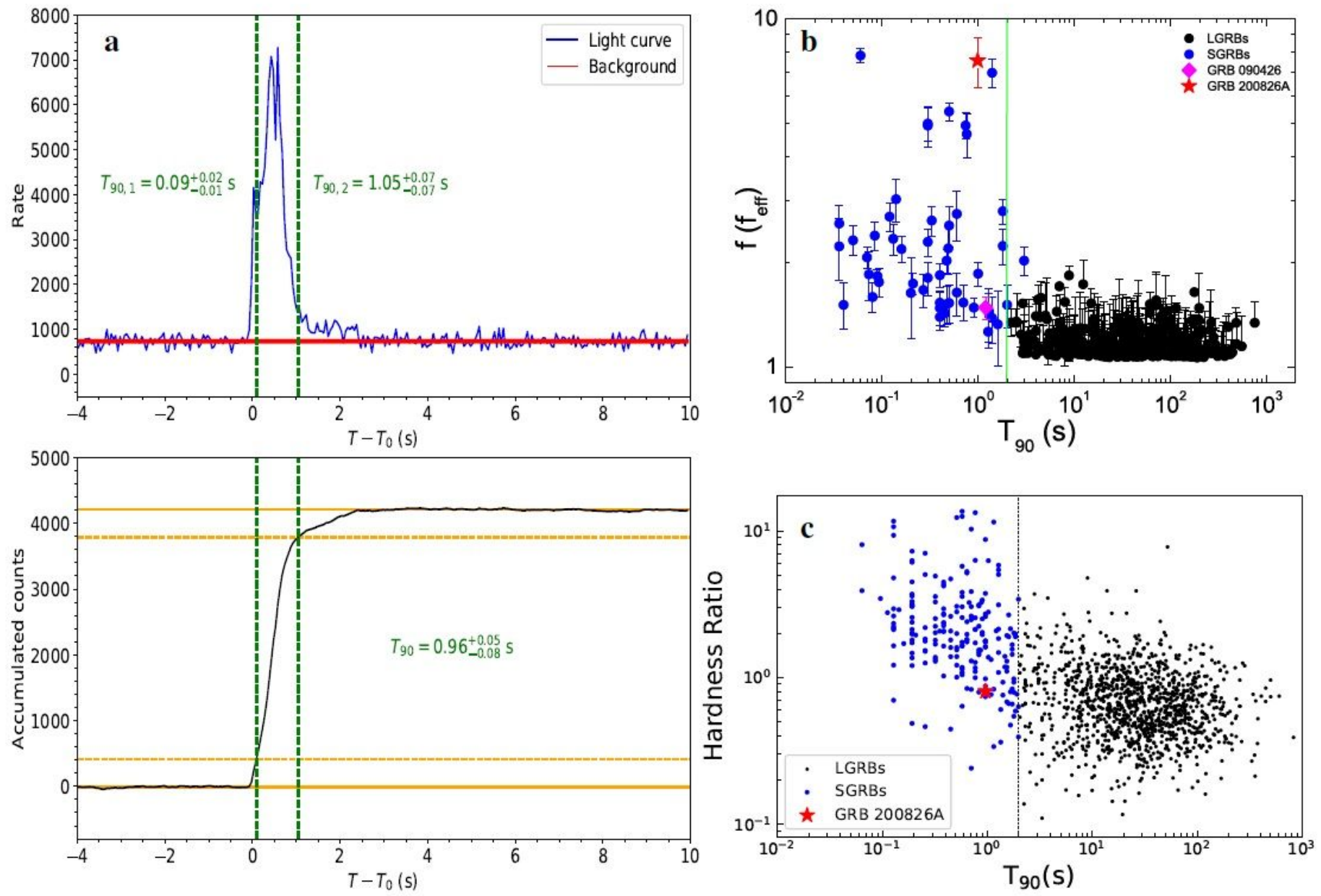

\section{Figure 1}

Temporal properties of GRB 200826A. a, T90 calculation : The upper panel shows the light curve and background flux level with blue and red solid lines, respectively. The accumulated counts as a function of time are shown with the black curve in the lower panel. The orange horizontal dashed (solid) lines are drawn at $5 \%(0 \%)$ and $95 \%(100 \%)$ of the total accumulated counts. The T90 interval is marked by the green vertical dashed lines. b, T90-f(feff) plot, where feff is the effective $f$ parameter when a long GRB becomes a disguised short GRB by arbitrarily lowering its flux level. GRB 200826A is highlighted by the red solid star. Blue circles correspond to $f$ parameter values of short GRBs, black circles mark feff values of long GRBs and green vertical line is the division line at 2 s. c, GRB 200826A on the T90-HR diagram : Blue and black points represent the short and long GRBs, respectively; red solid star marks GRB 200826A; and the black dashed line separate long and short GRBs at $2 \mathrm{~s}$. 

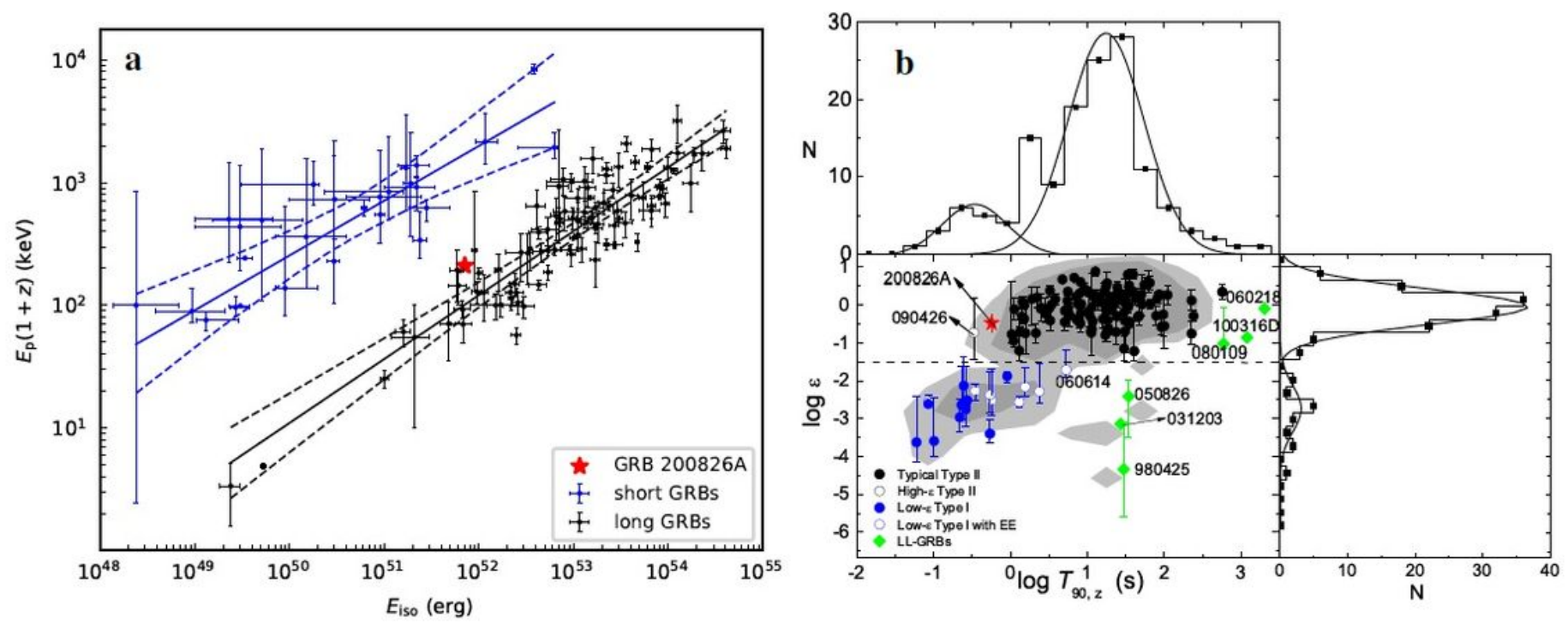

Figure 2

GRB 200826A in energy-related correlations. a, Ep,z - Ey,iso correlation diagram for short and long GRBs. Dashed borderlines show the $3 \sigma$ regions for each correlation. $b$, One and two dimensional distributions of GRB samples in the $\nabla-T 90$ space. Blue and black solid circles represent the Type I and Type II GRB

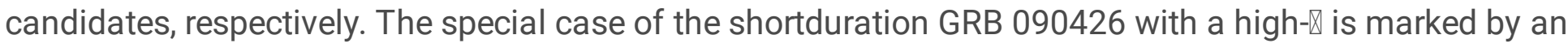
open black circle. Green diamonds denote the nearby low-luminosity long GRBs. Probability contours of Type I and II GRB clusters are also displayed in the grey regions (see Methods). The dashed horizontal line is the $\nabla=0.03$ division line. All error bars represent $1-\sigma$ uncertainties. One can see that GRB 200826A comfortably falls in the Type II region. 

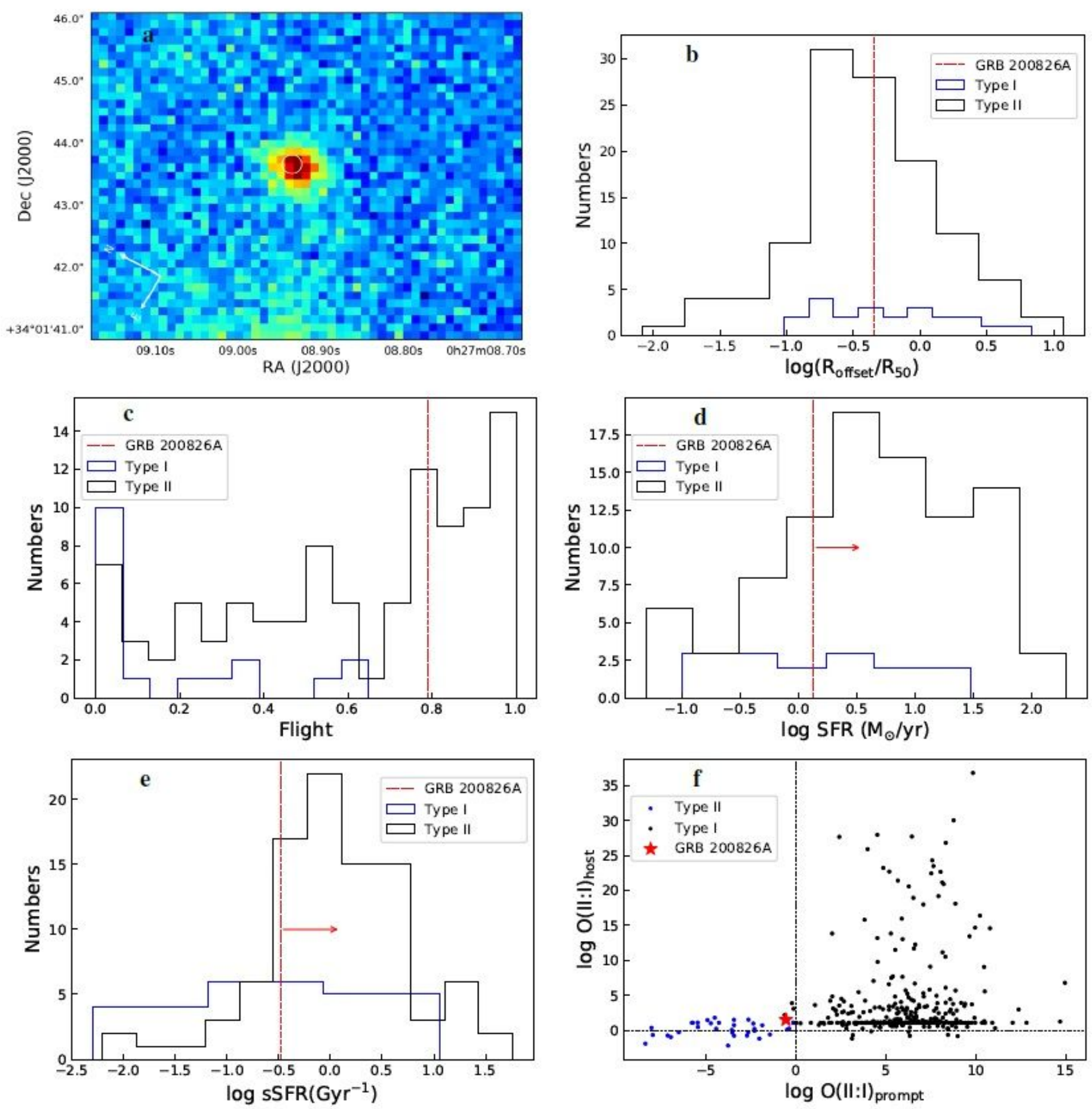

Figure 3

Host properties of GRB 200826A. a, The Gran Telescopio CANARIAS (GTC) Image of GRB 200826A's host galaxy. $b$, Normalized offset ( $r=$ Roff/R50) distributions of our samples. $c$, Distributions of cumulative light fraction Flight. d, Star formation rate (SFR) of the host galaxies. e, specific star formation rate (sSFR; the ratio between SFR and stellar mass of host galaxy M) of the host galaxies. $\mathrm{f}$, Posterior odds $\mathrm{O}$ (II:I) of prompt emission and host properties. GRB 200826A is marked with a red star. In b, c, d, and e, the black and blue histograms are for Type I and Type II GRBs, respectively. The vertical dashed red lines represent the location of GRB 200826A. 